\title{
Study on the Cure Rate of Invisible Headgear Orthodontic Opening Deep Occlusion of Anterior Teeth
}

\author{
Longfeng Wang \\ Nanjing Xinjiekou Dental Clinic, Nanjing 210000, Jiangsu Province, China
}

\begin{abstract}
[Abstract] Objective: To explore the cure rate of bracketless invisible headgear orthodontic for opening deep occlusion of anterior teeth. Methods: 10 cases with deep occlusion of anterior teeth were selected. All of the patients were admitted to our hospital from January 2017 to January 2020. All selected patients received bracketless invisible headgear orthodontic treatment. We took CBCT (cone beam CT) before and after orthodontics) lateral cranial radiographs and curved tomographic radiographs to analyze the effect of orthodontic treatment in 10 patients. Results: The changes of 10 patients before and after the orthodontic treatment were not significant in U1-SN, U6-SN, MP-FH $(\mathrm{P}>0.05)$; the U1-PP of the patients all decreased by $3 \sim 5 \mathrm{~mm}$. Compared with the effect before orthodontic treatment, the difference is significance $(\mathrm{P}<0.05)$; The tooth loosening didn't occur obviously in 10 patients. And 2 patients $(20.00 \%)$ had mild root resorption in the anterior teeth. Conclusion: The effect of invisible headgear orthodontic treatment without brackets is significant in orthodontic treatment of patients with deep occlusion of anterior teeth. The pressure of the upper anterior teeth can reach as low as $3 \sim 5 \mathrm{~mm}$. The cure rate of deep occlusion of open anterior teeth is high. The orthodontic method is comfortable, with beautiful appearance, which is easier to be accepted by patients.
\end{abstract}

Key words: Deep occlusion of anterior teeth; Depressed anterior teeth; Bracketless invisible orthodontic

Publication date: May, 2021; Publication online: 31 May, 2021

*Corresponding author: Longfeng Wang, longfengwang15@,163.com

\section{Introduction}

If the vertical distance between the upper anterior teeth covering the labial surface of the lower anterior teeth is too long, that is deep occlusion. It is a common malocclusion. Deep occlusion often coexists with deep overjet in patients with orthodontics. If we want to achieve the ideal orthodontic effect, the deep occlusion must be treated first to lay as the pre-requisite for subsequent treatment ${ }^{[1]}$. In addition, the anterior teeth are prone to loosening and displacement due to trauma in the treatment of patients with upper anterior tooth loss and defect. Deep occlusion of the anterior teeth can lead to problems such as insufficient space for the anterior teeth. Therefore, we need to conduct deep occlusion orthodontic for treating these patients. Bracketless invisible orthodontic has many advantages as concealment and beauty, which is favored by the majority of patients. This technology is widely used in the deep occlusion orthodontic treatment of the anterior teeth ${ }^{[2]}$. This article will explore the cure rate of invisible headgear orthodontic to open anterior deep occlusion.

\section{Materials and methods}

\subsection{Basic information}

We selected 10 patients with deep occlusion of the anterior teeth as the research objects, all of which were admitted to our hospital from January 2017 to January 2020. They are aged 18-41 years old, with the average age as $(29.68 \pm 2.13)$ years old. The male to female ratio is 4:6. 2 cases are Class II patients. 8 cases are Class I patients. 10 patients were with 
mild to moderate dental crowding, and deep occlusion of moderate and above degree. The selected patients obey well to the suggestions, doing regular oral maintenance. They are not pregnant, without any systemic diseases. And they have good general conditions. Patients with poor coordination, combined with dental pulp disease, periodontal disease and temporomandibular joint disease have been excluded. The selected patients voluntarily joined in the group, understanding the purpose and content of this study. Then they signed an informed consent form. This study was approved by the Medical Ethics Committee.

\subsection{Methods}

We allow the patient to undergo various examinations before orthodontic treatment to confirm that the patient's teeth can be corrected by invisible orthodontic technology. According to the different situation, we gave the patients the basic periodontal treatment such as root planing, root curettage, and supragingival cleansing. We actively communicate with the patient to understand the expected treatment effect and inner thoughts of the patients. We used Invisalign bracketless invisible headgear (produced by Align, USA), with the help of lateral cranial radiographs, oral panoramic photos and other imaging data, diagnosis and treatment records. That is how we can determine the occlusal relationship and level the spee curve. We depressed the front teeth and bicuspid to place rectangular attachments in the tooth are. We also lowered the canines and incisors. The Orthodontics Center uses 3D scanning technology to obtain and three-dimensionally reconstruct the dental and jaw plaster model. We make an invisible orthodontic device ${ }^{[3]}$. According to the treatment strategy, the doctor is responsible for bonding the accessories, instructing the patient to put on the orthosis correctly and in order, and instructing the patient to wear the invisible orthosis for no less than 20 hours a day. They should remove the orthosis when eating, maintaining oral hygiene with regularly follow up. After wearing for 1 month, the doctor should check the patient's orthodontic conditions, observing whether the accessories fall off, and adjusting the orthodontic plan according to the situation of the patient. If the orthosis is worn well and in close contact with the teeth, the patients can be reviewed every 2 months [4].

\subsection{Observation indicators}

CBCT (cone beam CT) lateral skull and curved tomography were taken before and after orthodontic. We recorded MP-FH (mandibular plane angle), U6-SN (maxillary first molar-anterior skull base plane angle), U1-SN (maxillary central incisor-anterior skull base plane angle), and U1-PP (maxillary central incisor-palatine plane distance). The method of Malmgren and Levander was used to evaluate the root resorption of patients ${ }^{[4]}$. The orthodontic effect of 10 patients was evaluated. Ineffective: the patient's chewing function and language function are limited. The deep occlusion is not significantly improved. The teeth are not neatly arranged. Effective: the patients with deep overjet is significantly recovered. The teeth are neatly arranged. The dentition changes significantly without any other temporomandibular joint problems or periodontitis disease. The patient's chewing function and language function were not affected.

\subsection{Statistical methods}

The new version of SPSS23.0 was used to analyze the collected data. When $\mathrm{P}<0.05$, it indicates that the comparison between the data is meaningful.

\section{Results}

\subsection{Depression of upper anterior teeth before and after orthodontic treatment}

Changes of U1-SN, U6-SN, MP-FH of 10 patients are not significant before and after orthodontic treatment $(\mathrm{P}>0.05)$; U1-PP of all patients decreased by $3 \sim 5 \mathrm{~mm}$. Compared with the condition before orthodontic, the difference was significant $(\mathrm{P}<0.05)$. It means that the upper anterior teeth are depressed. See Table 1 for details.

Table 1. Comparison of the occlusion situation of the upper anterior teeth before and after orthodontic treatment $( \pm \mathrm{s}, \mathrm{n}=10)$

\begin{tabular}{ccccc}
\hline & Before treatment & After treatment & t & P \\
\hline MP-FH $\left(^{\circ}\right)$ & $30.23 \pm 2.52$ & $29.61 \pm 2.45$ & 0.5578 & 0.5838 \\
U6-SN $\left(^{\circ}\right)$ & $33.52 \pm 2.48$ & $33.64 \pm 2.70$ & 0.1035 & 0.9187 \\
U1-SN $\left(^{\circ}\right)$ & $97.29 \pm 4.19$ & $98.68 \pm 4.25$ & 0.7365 & 0.4709 \\
U1-PP $(\mathrm{mm})$ & $25.69 \pm 1.26$ & $22.53 \pm 0.97$ & 6.2843 & 0.0000 \\
\hline
\end{tabular}




\subsection{Root resorption}

After orthodontic treatment, none of the 10 patients had obvious tooth loosening. 2 patients $(20.00 \%)$ had mild root resorption of the anterior teeth. The root length did not change significantly. It showed that the root tips were slightly rounded.

\subsection{Orthodontic effect}

The orthodontic effect of 10 patients with deep occlusion was satisfying, with an effective rate as $100 \%(10 / 10)$. Typical case: a 22-year-old male patient consulted in the implant department for anterior tooth trauma restoration. He was recommended to perform deep occlusion orthodontic treatment first. And then we conducted implantation and restoration. He was diagnosed as Class I, using Invisalign bracketless invisible headgear. We flattened Spee's curve, moderately adducting the upper incisors, and deglazing the adjacent surfaces of the upper anterior teeth. After the patient had worn 34 sets of appliances, the right upper central incisor had a proper gap. The molars and the canines were in a neutral relationship. And normal occlusion coverage was obtained. The root resorption of the upper anterior teeth was not obvious. After implant restoration treatment, the anterior teeth recovered their function and beauty.

\section{Discussion}

The aesthetics of teeth directly affects the overall appearance of a person's face. With the improvement of living standards, people are paying more and more attention to this. The proportion of patients who received orthodontic treatment is increasing. The introduction of bracketless invisible orthodontic technology effectively overcomes the shortcomings of traditional orthodontic technology in terms of comfort, aesthetics and ease of wearing. Depressing the anterior teeth or raising the posterior teeth is the main mechanism of deep occlusion orthodontic. In the past, implants, segmental arches, and versatile arch anchorage were mainly used to improve deep occlusion. Traditional orthodontic methods would have a negative impact on the patient's oral health. And it is not comfortable, which is easy to cause root atrophy, gum bleeding, etc. It will affect the patient's tooth aesthetics and chewing function to different degrees. The bracketless invisible appliance has the advantages that it is comfortable, beautiful and convenient to clean. The appliance is almost completely invisible, without arch wires, brackets, etc. So it has little irritation to the periodontal tissue. The patient won't feel any discomfort The results of this study showed that the change in U1-SN, U6-SN, and MP-FH of 10 patients before and after the orthodontic treatment is not significant $(\mathrm{P}>0.05)$; the U1-PP of the patients all decreased by $3 \sim 5 \mathrm{~mm}$. Compared with that before the orthodontic, the difference was statistically significant $(\mathrm{P}<0.05) ; 10$ patients don't have obvious tooth loosening, while 2 patients $(20.00 \%)$ had mild root resorption in the front teeth.

The invisible orthodontic technology has the following advantages in the anterior deep occlusion orthodontic: First, when the anterior teeth are depressed, the force of traditional fixed appliance acts on the labial side of the tooth impedance center. The movement of lips to a certain degree doesn't matter much for the patients with straight anterior teeth or tilted tongue. But for patients with more tilted lips of the front teeth, this method is inappropriate for the movement brought by the method of depressing the front teeth. The invisible appliance covers the incisal end and the lip and tongue side of the crown. The corrective force can depress the anterior teeth as a whole without the lip inclination of the teeth. If the patient's dentition is spaced, the invisible appliance can also depress and adduct the anterior teeth ${ }^{[5]}$. In addition, invisible orthodontic in deep occlusion treatment, we will depress the canines at the beginning, then depress the incisors. And then we achieve the stepwise depressing of the anterior teeth, which end up with a good orthodontic effect. Secondly, the bracketless invisible headgear technology, as the leading technology in orthodontic treatment, uses three-dimensional image technology to simulate the movement of the patient's teeth during the deep occlusion orthodontic treatment. We can effectively correct the deformity of the teeth by wearing transparent aligners. Compared with traditional fixed orthoses, it is more nice-looking and comfortable. We can visually predict the treatment results, which is convenient for patients to use. We can use it to assist other treatments ${ }^{[6]}$. It should be noted that studies have pointed out that there is still a risk of root resorption when the invisible appliance cure deep occlusion. And its severity cannot be reduced ${ }^{[7]}$. Depressing the teeth will lead to different degrees of root resorption in the process of traditional fixed orthodontics. Invisible headgear orthodontics also adducts the teeth, while the teeth are depressed. So, this problem cannot be completely avoided ${ }^{[8]}$. There are 2 patients with mild root resorption in this study, 
which is similar to domestic and foreign studies. However, this study is limited by time and sample size, which cannot fully solve the problem.

To sum up, the effect of bracketless invisible headgear orthodontic is significant in the orthodontic treatment of patients with deep occlusion of anterior teeth. The patient's upper anterior teeth can be depressed reaching by $3 \sim 5 \mathrm{~mm}$. The cure rate of deep occlusion of the anterior teeth is relatively higher, which is more comfortable and beautiful and is easier to be accepted by patients.

\section{References}

[1] Pan XG. Application of bracketless invisible orthodontic treatment in periodontal disease patients $[\mathrm{J}]$. Chinese Journal of Stomatology, 2020, 55(08): 546-550.

[2] Shi D. Observation of the clinical effect of invisible orthodontics without brackets and the study of its influence on periodontal state [J]. Journal of Clinical Stomatology, 2020, 36(279(01)): 47-50.

[3] Zhou L, Wang YM, Zhang L, et al. Invisible functional appliance for orthodontic of class II malocclusion in adolescents $[\mathrm{J}]$. West China Journal of Stomatology, 2019, (3): 236-241.

[4] Xu X. Study on the effect of three bonding methods for bracketless invisible appliance attachment $[\mathrm{J}]$. Chinese Physician Journal, 2018, 20(002): 281-283.

[5] Zhai MB, Sun Y, Guo JH, et al. Comparison of orthodontic treatment between malocclusion and traditional fixed orthodontics in children with malocclusion [J]. Journal of Hebei Medical University, 2019, 47(12): 25-29.

[6] Zhou XR, Feng H, Deng LH, et al. The clinical application effect analysis of bracketless invisible orthodontic technology $[\mathrm{J}]$. Chinese Journal of Women and Children Health Research, 2017, 28(S3): 425-426.

[7] Wang B. The application and advantage analysis of bracketless invisible orthodontic technology in adult orthodontics $[\mathrm{J}]$. Electronic Journal of General Stomatology, 2018, 5(020): 55,57.

[8] Ying G, Yan X. Bracketless invisible headgear orthodontic treatment and high quality nursing methods [J]. Chinese Community Doctors, 2018, (1): 10-11. 